ORIGINAL ARTICLE

\title{
Patellar tendinosis as an adaptive process: a new hypothesis
}

\author{
B Hamilton, C Purdam
}

Br J Sports Med 2004;38:758-761. doi: 10.1136/bjsm.2003.005157

Background: Patellar tendinosis (PT), or "jumper's knee" is a common condition in athletes participating in jumping sports, and is characterised by proximal patellar tendon pain and focal tenderness to palpation. Hypoechoic lesions observed in the proximal patellar tendon associated with the tendinosis are typically described as being a result of degenerative change or "failed healing". We propose a new

See end of article for authors' affiliations

.....................

Correspondence to: Dr B Hamilton, Australian Institute of Sport, Leverrier Crescent, Bruce, ACT Australia; bruce.hamilton@ eis2win.co.uk

Accepted 28 October 2003 model for the development of the hypoechoic lesion observed in PT, in which the aetiology is an adaptive response to differential forces within the tendon.

Methods: We assessed the clinical, histopathological, and biomechanical literature surrounding the patellar tendon and integrated this with research into the response of tendons to differential forces.

Results and conclusions: We propose that the hypoechoic lesion commonly described in PT is the result of adaptation or partial adaptation of the proximal patellar tendon to a compressive load. We postulate that the biomechanics of the patellar-patellar tendon interface creates this compressive environment. Secondary failure of the surrounding tensile adapted tendon tissue may result in tissue overload and failure, with resultant stimulation of nociceptors. We believe that this "adaptive model" of patellar tendinosis is consistent with the clinical and histological findings.
$\mathrm{T}$ endinosis is a term that was first used in the 1940s by German researchers. Its current use refers to tendon degeneration (or failed healing), with or without symptoms and without clinical or histological signs of inflammation. ${ }^{12}$ Patellar tendinosis (PT) is one of the most common of the chronic tendinopathies, with a high morbidity in elite jumping athletes, ${ }^{3}$ and is the preferred term for the histopathological syndrome first described as "jumper's knee" by Blazina in 1973. ${ }^{4}$ The condition displays varying degrees of both incapacitation and imaging abnormality. ${ }^{135}$ Symptomatic PT is characterised by proximal patellar tendon pain in jumping athletes, with focal tenderness to palpation. ${ }^{6}$ PT is common in elite athletes participating in jumping sports such as basketball and volleyball. ${ }^{378}$ Lian et al found that $53 \%$ of their elite volleyball players had symptoms of $\mathrm{PT}^{3}$ with symptoms ranging from mild to disabling. ${ }^{8}$ Despite a recent increase in literature regarding the clinical, radiological, and pathophysiological nature of this disorder, the exact pathoaetiological processes involved in the development of PT remain unclear. ${ }^{9}$ There are numerous inconsistencies in the current degenerative or failed healing approach to tendinosis, ${ }^{9}$ and there is little support for an inflammatory aetiology. ${ }^{10}$ Based on clinical observation, basic science, in vitro and in vivo research, and histological studies reviewed, we propose that the process of tendinosis is not degenerative, but rather an adaptive response to differential forces within tendons.

\section{CLINICAL ASPECTS}

Clinical diagnosis of patellar tendinosis involves eliciting a history of proximal patellar tendon pain with associated tenderness on palpation. Cook et $a l^{11}$ assessed the reliability, sensitivity, and specificity of palpation in the diagnosis of PT. They concluded that in "at risk", asymptomatic tendons, palpation alone is not a useful predictor of the presence of an ultrasonographic lesion, and that elite heavily trained young athletes may have mild tendon soreness without the presence of an ultrasonographic abnormality. ${ }^{11}$

Although confirmation of a clinical diagnosis of PT has traditionally been based around the presence of an ultrasonographically visualised hypoechoic lesion in the proximal patellar tendon, there is a poor correlation between hypoechogenicity and symptoms. ${ }^{7}$ Indeed, longitudinal studies have suggested that sonographic hypoechoic lesions can both pre-date and post-date the symptoms of PT. ${ }^{7}$ Cook et al found that $22 \%$ of asymptomatic elite sports men and women had hypoechoic lesions at the proximal pole of the patellar tendon. The authors concluded that the presence of an ultrasonographic hypoechoic lesion in an elite athlete with anterior knee pain does not confirm a diagnosis of PT and that hypoechoic lesions may commonly be asymptomatic. By contrast, the ultrasonographic variations observed in PT have been shown to correlate strongly with histological abnormalities. ${ }^{12}$

\section{HISTOPATHOLOGY}

Limited histopathological studies have been performed on tendons affected by PT.

Features of PT include separation of collagen fibres, increased mucoid ground substance, plump tenocytes, fibrocartilagenous metaplasia, and cellular and capillary proliferation. $^{8}{ }^{12}$ Proliferation of neural tissue ${ }^{13}$ and elevated levels of glutamate and glutamate receptors have also been reported. ${ }^{10}$ The separation of collagen fibres and increased mucoid ground substance is classically described as degenerative change, and is observed most commonly at the posterior aspect of the proximal patellar tendon. ${ }^{6}$

Khan et a ${ }^{12}$ histologically examined 28 patellar tendons at open tenotomy for PT. Patients had an average of 35 months of symptoms, recalcitrant to conservative treatment. Histological findings in the patients were compared with 39 cadaver tendons. Findings consistently revealed a loss of normal dense linear collagen organization, with increased mucoid ground substance. Tenocytes were plump and chondroid in nature. In addition, there was cellular proliferation within the tendon, with prominent capillary proliferation. Both the cellular and the vascular proliferation ended at the area of increased mucoid ground substance, which was consistently found at the proximal pole of the patellar tendon. No acute inflammatory cells were found. By comparison, 34 of the cadaver tendons

Abbreviations: PGE2, prostaglandin E2; PT, patellar tendinosis 
showed a dense homogeneous collagen pattern, inconspicuous tenocytes, absent stainable ground substance, and no fibroblastic or capillary proliferation. Interestingly, three of the cadaver tendons showed similar changes to those found in the patients' tendons. Similar findings have been described in other papers. ${ }^{8} 1415$

Recently, Sanchis-Alfonso et al ${ }^{13}$ evaluated 17 tendons of individuals who had experienced more than 6 months of Blazina stage III patellar tendinopathy. The patients had an average of 13 months of symptoms at the time of tenotomy. The researchers found a histological pattern of "nerve sprouting" in the osteotendinous zone of the proximal patellar tendon, with some features of neuromatous change. They also found myelinated nerve endings within the walls of arterial vessels. This feature was particularly prominent in the fat pad adjacent to the proximal patellar tendon. They suggest that this vascular innervation may contribute to the pain of PT through the release of neurotransmitters such as substance P. Unfortunately, there was no control group with which to compare these findings.

Alfredson et $a l^{10}$ using an in vivo microdialysis technique, assessed the levels of glutamate and prostaglandin E2 (PGE2) in jumper's knee. They found significantly elevated levels of glutamate, but no difference in the levels of PGE2 when comparing the patellar tendon of symptomatic subjects with controls. In addition, the authors reported glutamate receptors in the region of nerve structures, but inflammatory cells were absent. Alfredson et al propose a role for glutamate in the production of pain in tendon disorders. ${ }^{10}$

A common feature of many of the histological papers is the lack of clarity on the source of the tissue being examined. Khan et $a l^{12}$ in their landmark paper quite clearly delineate areas of hypocellularity and hypercellularity, along with areas of increased vascularisation and ground substance deposition. However, most histological papers reviewed fail to clearly delineate the particular region of the tendon in which the pathological variations are present and this makes accurate interpretation difficult. However, it does appear that the area corresponding to the ultrasonographic hypoechogenicity consistently contains tenocytes with plump rounded nuclei. ${ }^{12}{ }^{16}$ Ground substance consisting of large molecular weight proteoglycans with associated increased water content abound. Collagen is of a smaller diameter and irregularly distributed. ${ }^{2}$ In the tissue surrounding the hypoechoic tissue, it appears that the tenocyte number may be increased. There may occasionally be lymphocytes. Both proliferation of capillaries and neuronal sprouting, ceasing at the edge of the hypoechoic zone, have been described. ${ }^{13} 1718$

\section{AETIOLOGY}

Classic theories appear to consider the aetiological process involved in both the area of hypoechogenicity and the surrounding tissue to be the same. In general, mechanical models for the pathogenesis of PT consider that tensile failure promotes healing and tensile adaptation, with repeated tensile insult preventing successful healing and producing the accumulation of (so-called) degenerative tissue. It has been proposed that the residual overloaded collagen fibres may be the source of pain. ${ }^{9}{ }^{19}$ Recent work suggests that both glutamate and neuronal sprouting may be involved in the generation of pain in chronic tendon conditions. ${ }^{1013}$

\section{WEAKNESSES IN TRADITIONAL THEORIES}

We have identified a number of weaknesses in a tensile failure, failed healing, degenerative model for the formation of patellar tendinosis:

- If tensile failure of tissues is the precursor to the formation of the hypoechoic region of tendinopathy, why is the lesion commonly found in pain free knees? It seems unlikely that either failed healing or degenerative changes should occur without any pain, especially when it is proposed that partially torn tendon may be the source of pain. ${ }^{9}$ We consider it more likely that a painless process of tendon change is occurring, followed by the reaching of a critical tensile load failure point, stimulating nociceptors.

- If tensile failure results in the accumulation of degenerative tissue with no tension resistant properties, the remaining functional tendon tissue will be under increased tensile load per unit volume. The natural progression of this, if overloading of the tendon continues, should be a continued acceleration of the formation of degenerative tissue, until the entire tendon is degenerative. This has not been observed in longitudinal studies. ${ }^{7}$

- If the same process creating the hypoechoic tendinosis region is occurring in the surrounding tendon tissue, there should be a transitional zone where tissue is undergoing progressive degeneration. This has not been described to date in histopathological studies.

- Degenerative tendon areas are considered to reflect a "change in tissue from a higher to a lower or less functionally active form". ${ }^{19}$ Production of high molecular weight proteoglycans and type III collagen may reflect not a higher or lower degree of activity or function, but merely an altered function, ${ }^{20}$ as tendons are known to alter their morphology as a result of differential forces. ${ }^{21}$

- The degeneration of any tissue is not considered to be reversible. ${ }^{19}$ It is clear from imaging and clinical studies that PT may be reversed. ${ }^{1}$

- Ageing tendons undergo histological changes quite distinct from the so-called degenerative changes observed in PT. ${ }^{12} 22$

These weaknesses in the interpretation of histological and clinical findings have driven us to propose a new model for the formation of tendinosis, not based on a principally tensile failure model. We propose an alternative model for both the development of patellar tendinosis and the occurrence of pain.

\section{ADAPTIVE MODEL OF PATELLAR TENDINOSIS Proposal}

We propose that tensile tissue failure is not the precipitant for the formation of PT. Given the biomechanics of the patellar-patellar tendon interface, we postulate that a compressive force exists at the proximal posterior aspect of the patella tendon. This compressive force results in attempted histological adaptation to compressive loading, at the expense of tensile properties. This adaptive response may be pain free, and would explain the presence of hypoechoic lesions in asymptomatic subjects. With increasing size of the compression adapted tissue in the proximal posterior aspect of the patellar tendon, the remaining tensile adapted tissue becomes overloaded and may partially fail, stimulating both nociceptors and an increased cellular and humoral response. We believe that this "adaptive model" of patellar tendinosis is more consistent with clinical and histological findings than any of the current models.

\section{Response of tendons to compressive load}

In 1994, Robbins and Vogel ${ }^{23}$ illustrated that the nature of proteoglycans and collagen differed in areas of tendons that were subject to loads of either compression or tension. In those areas undergoing compression, high molecular weight proteoglycans (such as aggrecan) and type II collagen mRNA predominate. By contrast, in tendons undergoing tensional load, the major proteoglycans are typically the low molecular weight decorin, and the collagen fibres are predominantly of 
type $\mathrm{I}^{23}$ The authors conclude that the gene expression for collagen and proteoglycans within a tendon correlates with the mechanical environment in the tissue. Similarly, Robbins et $a l^{24}$ found that fetal tendons subject to a cyclical compressive load exhibited enhanced expression of the mRNA for aggrecan and biglycan (high molecular weight proteoglycans) as well as for transforming growth factor- $\beta$.

Vogel and Koob ${ }^{21}$ describe tendons undergoing a compressive load as histologically manifesting increased glycosaminoglycan content, loss of normal parallel collagen fibre deposition, increasingly rounded cells appearing in cartilage-like lacunae, and altered proteoglycan content. These changes were shown to be partially reversible over time and were felt to be adaptive, rather than degenerative. Similar histological changes have been described in the hypoechoic tissue of PT. ${ }^{12}$

\section{Compressive loads within the patellar tendon}

Leadbetter $^{19}$ considers that compressive forces to tendons may occur extrinsically at sites of pulleys and bony prominences, or intrinsically as a result of cyclical torque load. We believe that there exists in the patellar tendon the potential for compression of the proximal posterior patellar tendon fibres against the distal patella, in the region of the patella-patellar tendon interface.

Johnson $^{25}$ compared the MRI findings for 24 patellar tendons with a diagnosis of PT with matched asymptomatic control tendons. They found increased signal intensity in the proximal posterior aspect of 19 of the subject tendons, but in none of the asymptomatic controls. Based primarily upon the location of the lesion, they felt that impingement of the patellar tendon against the distal pole of the patella was most probably the explanation for the increased signal intensity observed. ${ }^{25}$ More recently, in order to assess the importance of impingement in the formation of PT, Schmid et al ${ }^{26}$ compared 19 symptomatic with 32 asymptomatic, but athletic, knees. Using open configuration MRI, sagittal images of the patellar tendon were obtained in 10 degree increments from full extension to 100 degrees of flexion, with the quadriceps muscle alternatively relaxed and contracted. They found that with a contracted quadriceps muscle the angle between the patella and the patellar tendon decreased from 157 to 145 degrees during flexion, with the maximum angulation occurring at between 50 and 70 degrees of knee flexion. As no significant difference was found between symptomatic and asymptomatic subjects with regard to the patella-patellar tendon angle, the authors concluded that PT cannot be caused by impingement of the patellar tendon against the distal patella. ${ }^{26}$ Interestingly, however, 23/32 of the asymptomatic subjects were found to have areas of increased signal intensity (consistent with PT) at the posterior aspect of the proximal patellar tendon.

We speculate that, based on the impingement model described by Johnson, ${ }^{25}$ and supported by the work of Schmid et al, ${ }^{26}$ evidence exists for a potential compressive force at the proximal posterior aspect of the patellar tendon during the first 60 degrees of flexion (fig 1).

\section{Adaptive model and the hypoechoic tendon}

Having identified a potential compressive force to the proximal posterior aspect of the patellar tendon, we propose that the histological changes observed in the hypoechoic region of the patellar tendon are consistent with an adaptive change to altered forces in this particular area of the tendon. This is a significant change from the current degenerative or failed healing models (which may indeed produce a similar histological outcome) proposed for the formation of tendinosis. An adaptive model accounts for the presence of asymptomatic "lesions" and their ability to resolve. It appears
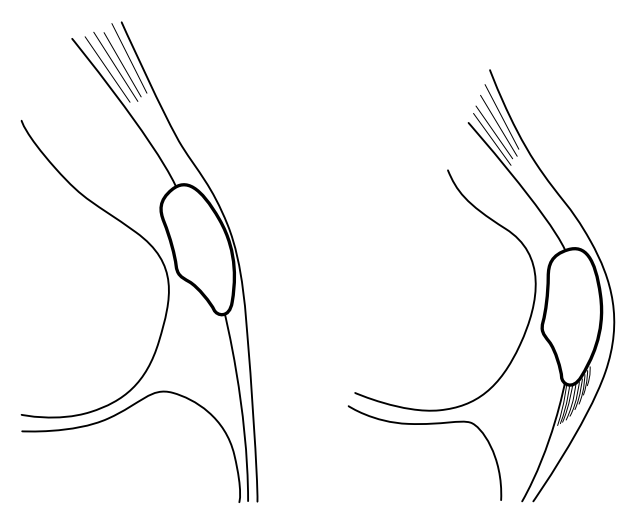

Figure 1 Proposed area of patellar tendon compression during knee flexion.

consistent with the histopathological findings, explains the dearth of inflammatory cells, and allows for the distinct changes found in an ageing tendon. An adaptive model to a localised compressive load accounts for the non-progressive nature of US monitored hypoechogenicity, which confounds models proposing tensile failure.

\section{Adaptive model and the presence of pain}

With the formation of altered tissue in the proximal posterior aspect of the patellar tendon, which is adapted, or partially adapted, to compressive forces, it is clear that the surrounding tendon tissue will be placed under increased tensile stress. It would appear likely that the ability of the surrounding tissue to withstand tensile load is the most important feature in the prevention of tendon pain, not the size of the hypoechoic area. Numerous papers have outlined the process of tissue failure secondary to tensile overload, ${ }^{9}{ }^{19}$ and we propose that as a result of compression adapted tissue no longer actively resisting tensile load, secondary failure of the surrounding tissue may occur. Failure of the surrounding tissue may explain the increase in cell number, angiogenesis, and neuronal sprouting observed in the area immediately adjacent to the hypoechoic tendinosis lesion. ${ }^{12}$ Recent papers have shown a relationship between increased vascularisation, neuronal sprouting, glutamate, and the presence of pain. ${ }^{1017182728}$

\section{Role of inflammation and inflammatory mediators}

While no inflammatory cells have been reported in PT, most samples are from chronically symptomatic tissue. Almekinders et al ${ }^{29}$ examined the effect of repetitive tensional load on tendon fibroblasts (tenocytes), and found that those fibroblasts subjected to repetitive strain produced significantly elevated levels of PGE2 compared with those undergoing no strain. This may suggest a link between tensional load and PT, as Fu et al ${ }^{30}$ have found elevated levels of PGE2 in PT. In contrast to these findings, Alfredson et $a l,{ }^{10}$ using an in vivo microdialysis technique, assessed the levels of glutamate, glutamate receptors, and PGE2 in chronic PT. They found significantly elevated levels of glutamate, but no elevation of PGE2 levels.

Notwithstanding the difficulties involved in these experimental techniques, if tenocytes under tensile strain produce elevated levels of PGE2, it is surprising that PGE2 levels are not elevated in microdialysis studies, ${ }^{10}$ given that the classical proposed aetiology for PT is tensile overload. We speculate that these findings may be better explained by a proximal, adaptive hypoechoic area, surrounded by an area of tensile overload. 


\section{WHAT IS ALREADY KNOWN ON THIS TOPIC}

- Patellar tendinosis or "jumper's knee" is a common condition affecting jumping athletes.

- Patellar tendinosis is a common problem in jumping athletes, but one in which the aetiology is yet to be clearly delineated.

- The exact aetiology of the condition is unclear with histopathological and biochemical studies yielding ambiguous results.

- Traditional imaging techniques are considered to provide little prognostic information, and treatment modalities continue to be based on experience rather than confirmed pathoaetiological processes.

- We postulate that the hypoechoic area observed in ultrasonographic studies may result from adaptation of tendon tissue to a compressive load in the proximal, posterior aspect of the patellar tendon.

- Further examination of the histopathology and differential forces applied to the patellar tendon is required.

\section{WHAT THIS STUDY ADDS}

- This paper attempts to rationalise studies from various disciplines into a single united theory.

- It provides a framework to stimulate future research and enhance the ongoing understanding and management of patellar tendinosis.

\section{CONCLUSION}

It is well recognised that tendon tissue morphologically adapts to variations in the forces being applied. While the histopathology of patella tendinosis has classically been described as "degenerative" or "failed healing", we postulate that the aetiology is an adaptive change secondary to differential compressive forces within a tendon. The remaining tendon is therefore placed under increasing tensile load, resulting in peripheral tissue failure, pain, and cellular proliferation. The development of this model is based primarily around dissatisfaction with the current theories of tendinosis aetiology. The major limitation to this proposal is the lack of hard evidence of the presence of compression forces in the proximal patellar tendon. We believe that the histological similarities between compressed tendon tissue and tendinosis tissue should be investigated. Assessment of the forces within the patellar tendon need to be further evaluated, as it seems unlikely that the entire tendon is subject to uniform forces throughout its range of motion. We propose this process as a hypothesis for future research.

\section{Authors' affiliations}

B Hamilton, Australian Institute of Sport, Leverrier Crescent, Bruce, ACT, Australia

C Purdam, Australian Institute of Sport, Leverrier Crescent, Bruce, ACT, Australia

Conflict of interest: none declared

\section{REFERENCES}

1 Cook JL, Khan KM, Harcourt PR, et al. Patellar tendon ultrasonography in asymptomatic active athletes reveals hypoechoic regions: a study of 320 tendons. Victorian Institute of Sport Tendon Study Group. Clin J Sport Med 1998;8:73-7

2 Khan KM, Cook JL, Bonar F, et al. Histopathology of common tendinopathies. Update and implications for clinical management. Sports Med 1999;27:393-408.

3 Lian O, Holen KJ, Engebretsen L, et al. Relationship between symptoms of jumper's knee and the ultrasound characteristics of the patellar tendon among high level male volleyball players. Scand J Med Sci Sports 1996;6:291-6.

4 Blazina ME, Kerlan RK, Jobe FW, et al. Jumper's knee. Orthop Clin North Am 1973;4:665-78.

5 Fredberg U, Bolvig L. Jumper's knee. Review of the literature. Scand J Med Sci Sports 1999:9:66-73.

6 King J, Cook J, Khan K, et al. Patellar Tendinopathy. Sports Med Arthroscopy Rev 2000;8:86-95.

7 Khan KM, Cook JL, Kiss ZS, et al. Patellar tendon ultrasonography and jumper's knee in female basketball players: a longitudinal study. Clin J Sport Med 1997;7:199-206.

8 Cook JL, Khan KM, Harcourt PR, et al. A cross sectional study of 100 athletes with jumper's knee managed conservatively and surgically. The Victorian Institute of Sport Tendon Study Group. Br J Sports Med 1997;31:332-6.

9 Khan K, Cook J. Overuse tendon injuries: where does the pain come from? Sports Med Arthroscopy Rev 2000;8:17-31.

10 Alfredson $\mathrm{H}$, Forsgren S, Thorsen $\mathrm{K}$, et al. In vivo microdialysis and immunohistochemical analyses of tendon tissue demonstrated high amounts of free glutamate and glutamate NMDAR1 receptors, but no signs of inflammation, in Jumper's knee. J Orthop Res 2001;19:881-6.

11 Cook JL, Khan KM, Kiss ZS, et al. Reproducibility and clinical utility of tendon palpation to detect patellar tendinopathy in young basketball players. Victorian Institute of Sport tendon study group. Br J Sports Med $2001 ; 35: 65-9$

12 Khan KM, Bonar F, Desmond PM, et al. Patellar tendinosis (jumper's knee): findings at histopathologic examination, US, and MR imaging. Victorian Institute of Sport Tendon Study Group. Radiology 1996;200:821-7.

13 Sanchis-Alfonso V, Rosello-Sastre E, Subias-Lopez A. Neuroanatomic basis for pain in patellar tendinosis ("jumper's knee"): a neuroimmunohistochemical study. Am J Knee Surg 2001;14:174-7.

14 Popp JE, Yu JS, Kaeding CC. Recalcitrant patellar tendinitis. Magnetic resonance imaging, histologic evaluation, and surgical treatment. Am J Sports Med 1997;25:218-22.

15 Jarvinen M, Jozsa L, Kannus $P$, et al. Histopathological findings in chronic tendon disorders. Scand J Med Sci Sports 1997;7:86-95.

16 Shalabi A, Kristoffersen-Wiberg M, Papadogiannakis N, et al. Dynamic contrast-enhanced MR imaging and histopathology in chronic achilles tendinosis. A longitudinal MR study of 15 patients. Acta Radiol 2002;43:198-206.

17 Ohberg L, Alfredson $\mathrm{H}$. Ultrasound guided sclerosis of neovessels in painful chronic Achilles tendinosis: pilot study of a new treatment. Br J Sports Med 2002;36:173-5; discussion 176-7.

18 Ohberg L, Lorentzon R, Alfredson H. Neovascularisation in Achilles tendons with painful tendinosis but not in normal tendons: an ultrasonographic investigation. Knee Surg Sports Traumatol Arthrosc 2001;9:233-8.

19 Leadbetter WB. Cell-matrix response in tendon injury. Clin Sports Med 1992;11:533-78.

20 Rolf CG, Fu BS, Pau A, et al. Increased cell proliferation and associated expression of PDGFRbeta causing hypercellularity in patellar tendinosis. Rheumatology (Oxford) 2001;40:256-61.

21 Vogel KG, Koob TJ. Structural specialization in tendons under compression. Int Rev Cytol 1989;115:267-93.

22 Tuite DJ, Renstrom PA, O'Brien M. The aging tendon. Scand J Med Sci Sports 1997;7:72-7

23 Robbins JR, Vogel KG. Regional expression of mRNA for proteoglycans and collagen in tendon. Eur J Cell Biol 1994;64:264-70.

24 Robbins JR, Evanko SP, Vogel KG. Mechanical loading and TGF-beta regulate proteoglycan synthesis in tendon. Arch Biochem Biophys 1997;342:203-11.

25 Johnson DP, Wakeley CJ, Watt I. Magnetic resonance imaging of patellar tendonitis. J Bone Joint Surg Br 1996;78:452-7.

26 Schmid MR, Hodler J, Cathrein P, et al. Is impingement the cause of jumper's knee? Dynamic and static magnetic resonance imaging of patellar tendinitis in an open-configuration system. Am J Sports Med 2002;30:388-95.

27 Weinberg EP, Adams MJ, Hollenberg GM. Color Doppler sonography of patellar tendinosis. Am J Roentgenol 1998;171:743-4.

28 Terslev L, Qvistgaard E, Torp-Pedersen S, et al. Ultrasound and Power Doppler findings in jumper's knee - preliminary observations. Eur J Ultrasound 2001;13:183-9.

29 Almekinders LC, Banes AJ, Ballenger CA. Effects of repetitive motion on human fibroblasts. Med Sci Sports Exerc 1993;25:603-7.

30 Fu SC, Wang W, Pau HM, et al. Increased expression of transforming growth factor-betal in patellar tendinosis. Clin Orthop 2002;400:174-83. 